\title{
THE MASS TO LIGHT RATIO OF MARKARIAN GALAXIES
}

\author{
John P. Huchra \\ Hale Observatories \\ California Institute of Technology \\ Carnegie Institution of Washington
}

Une estimation du rapport moyen masse sur luminosité des galaxies de Markarian est déterminée à partir de bonnes mesures de vitessespour des paires serrées de galaxies de Markarian. Une comparaison avec les données rassemblées par Turner pour des galaxies de champ montre que les galaxies de Markarian et les galaxies de champ ont le même rapport moyen masse sur luminosité. Le degré de regroupement en amas ou en paire des galaxies de Markarian est comparé avec celui trouvé pour les galaxies de champ. Les galaxies de Markarian ne montrent pas une tendance plus grande que les autres galaxies à se regrouper en paires.

As part of my study of the integrated properties of Markarian galaxies (Huchra 1976), it was interesting to determine their mean mass to light ratio. This interest was heightened by the suggestion of Heidmann and Kalloghlian (1973) - based on the rather poor velocity data available at the time - that Markarian galaxies have abnormally high mass to light ratios, or exist in unbound pairs.

The writer has collected and obtained velocity and separation data for 20 Markarian pairs and Markarian double galaxies with separations less than 8 arc-minutes. An estimate of the minimum system mass is made using Page's (1966) formula and

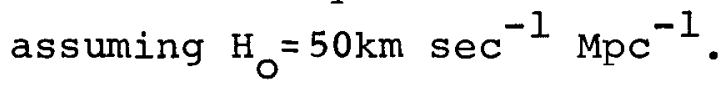

$$
\mathrm{M} / \mathrm{M}_{\odot} \geq 1350 \quad \mathrm{D} \mathrm{V}_{\mathrm{r}}(\Delta \mathrm{V})^{2} \text {. }
$$

The system luminosity is given by

$$
\mathrm{L} / \mathrm{L}_{\odot}=\operatorname{dex}\left[-0.4\left(\mathrm{M}_{\mathrm{pg}}-5.26\right]\right)
$$

$D$ is the separation in arc minutes, $\Delta \mathrm{V}$ is the velocity differ- 
ence, $V_{r}$ is the mean radial velocity, and $\mathrm{M}_{\mathrm{pg}}$ is the absolute photographic magnitude. Table 1 presents the parameters for the Markarian pairs and doubles.

TABLE 1

MARKARIAN PAIRS WITH COMPLETE RADIAL VELOCITY DATA

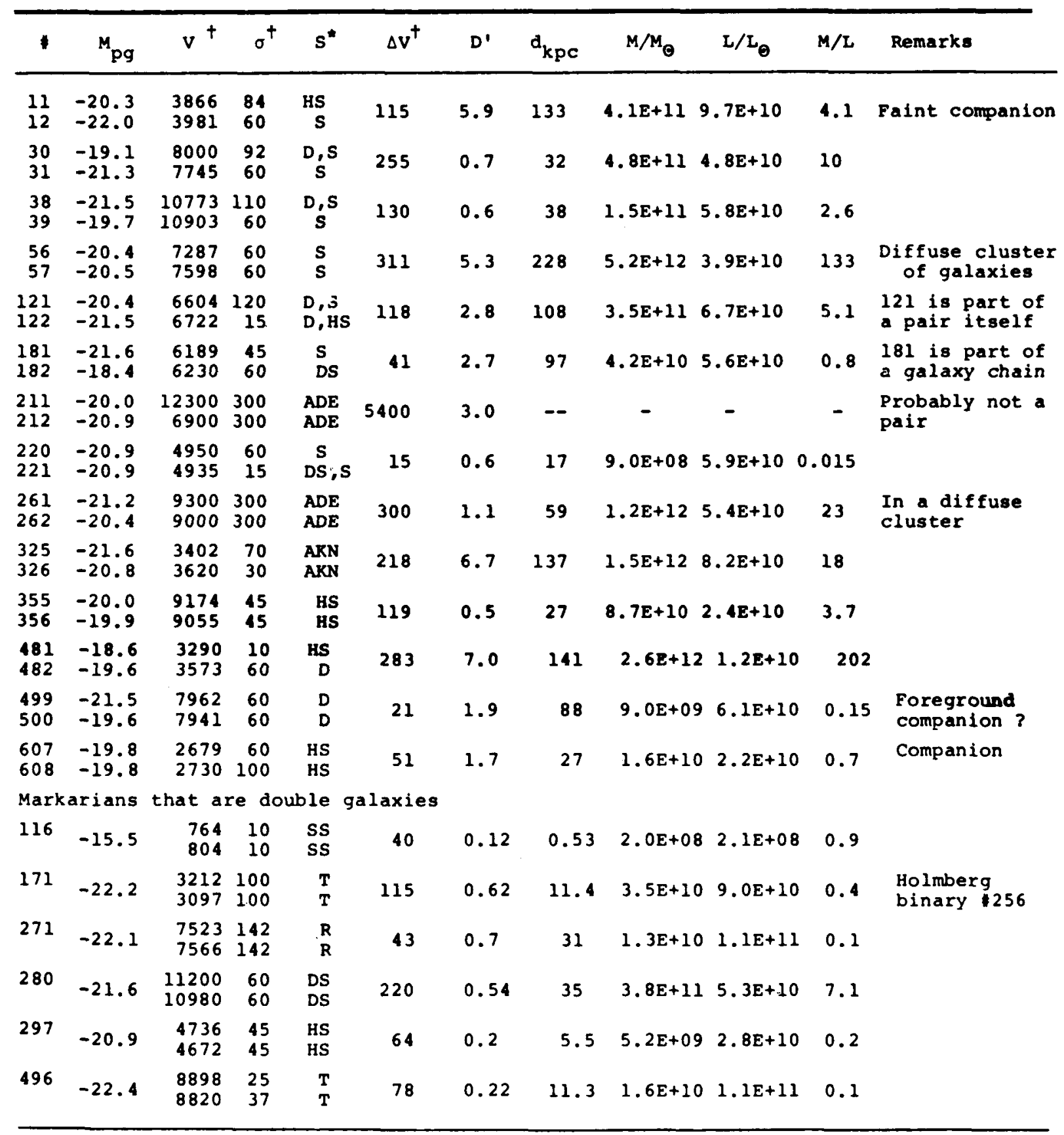

+ in $\mathrm{km} \mathrm{sec}-1$ 
Sources: HS Huchra and Sargent (1973, 1976)

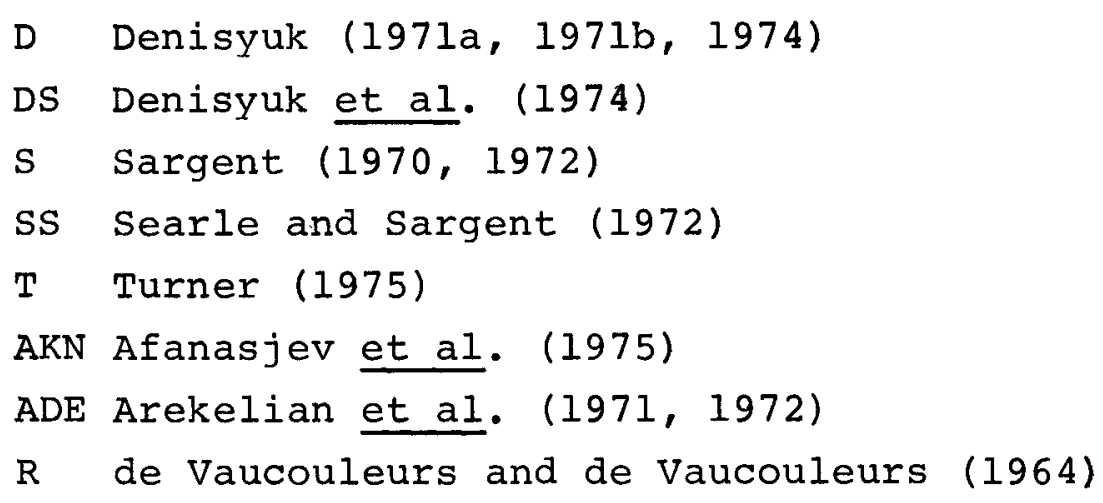

Markarians 211 and 212 are probably a chance superposition. The last column contains a description of the surrounding field with respect to the nearest neighbor parameter $\mathrm{x}=5$ (Turner 1975). Only one pair has a $\Delta V$ greater than $300 \mathrm{~km} \mathrm{sec}-1$ while seven pairs have additional galaxies associated with them or appear to be members of clusters.

By using Markarian-Markarian binaries, we can make an estimate of the mean mass to light ratio for Markarian galaxies as a class and compare it to estimates for field galaxy mass to light ratios. Turner (1975) has obtained data for a well defined sample of binary galaxies. Thirteen of our pairs or doubles (assuming that the resolved companion to $499 / 500$ is in the foreground) conform to Turner's "binary" separation and nearest neighbor cutoffs, but almost all of the systems have one component fainter than Turner's $M_{p g}=15.0$ cutoff. Does the limiting magnitude difference introduce selection effects? There are two competing processes - fainter galaxies are, on the average, farther away, so that a given projected separation will become a larger spatial separation, but it is easier to find pairs with smaller projected separations as the magnitude limit decreases. We checked the hypothesis that the Markarian and Turner binary galaxies represent a similar sample of spatial galaxy separations by using the Wilcoxon rank-sum test (Bury 1975, Noether 1967). This is a nonparametric test that compares two samplings of data distributions by ordering the combined sample and comparing the summed ranks of the smaller sample 
with the expected sum of a random sampling of that size. The test shows that there is no significant difference between the two samples - they are drawn from the same parent population and can be used to compare mass to light ratios. Figures 1 and 2 show the distributions of physical separations and velocity differences for Turner's field galaxy pairs and the Markarian pairs.

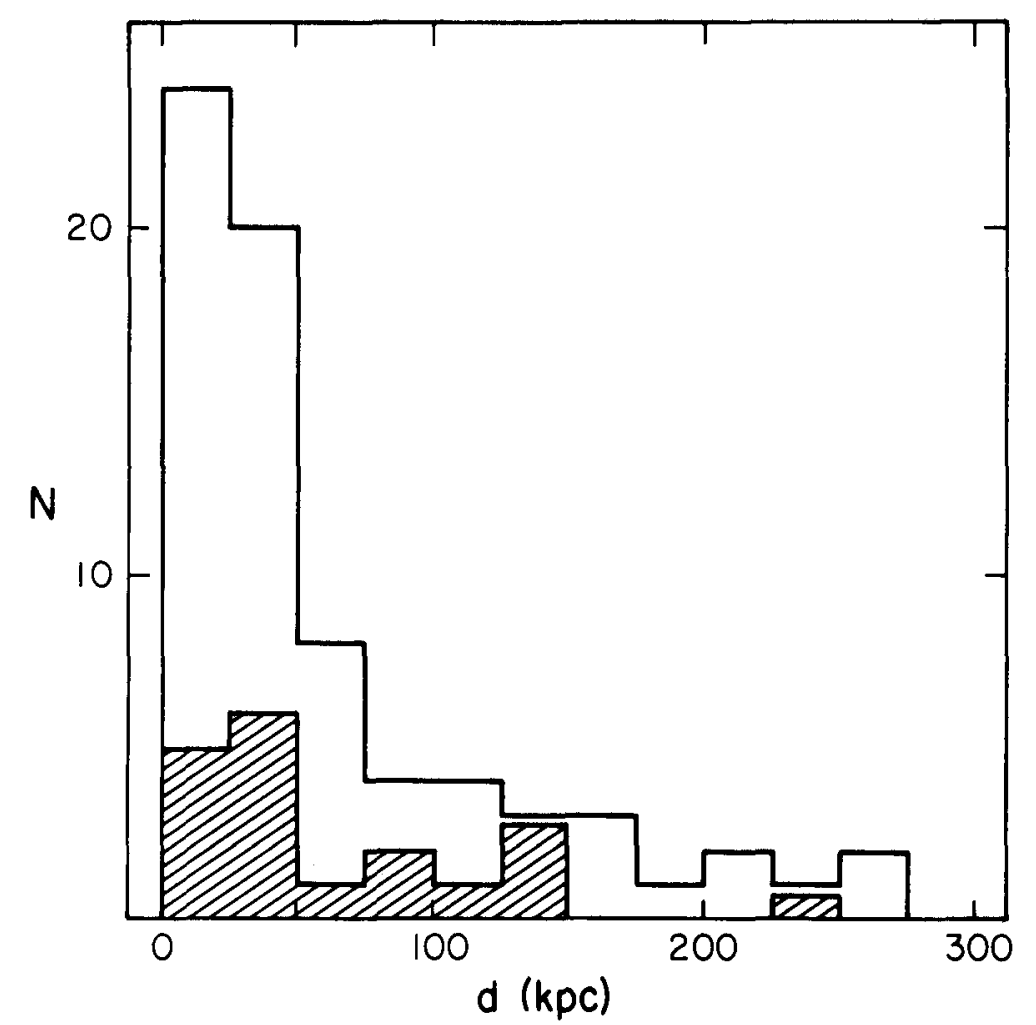

Figure 1. Distributions of separations (in kpc) for Turner's (1975) field galaxy pairs and Markarian pairs (shaded). 


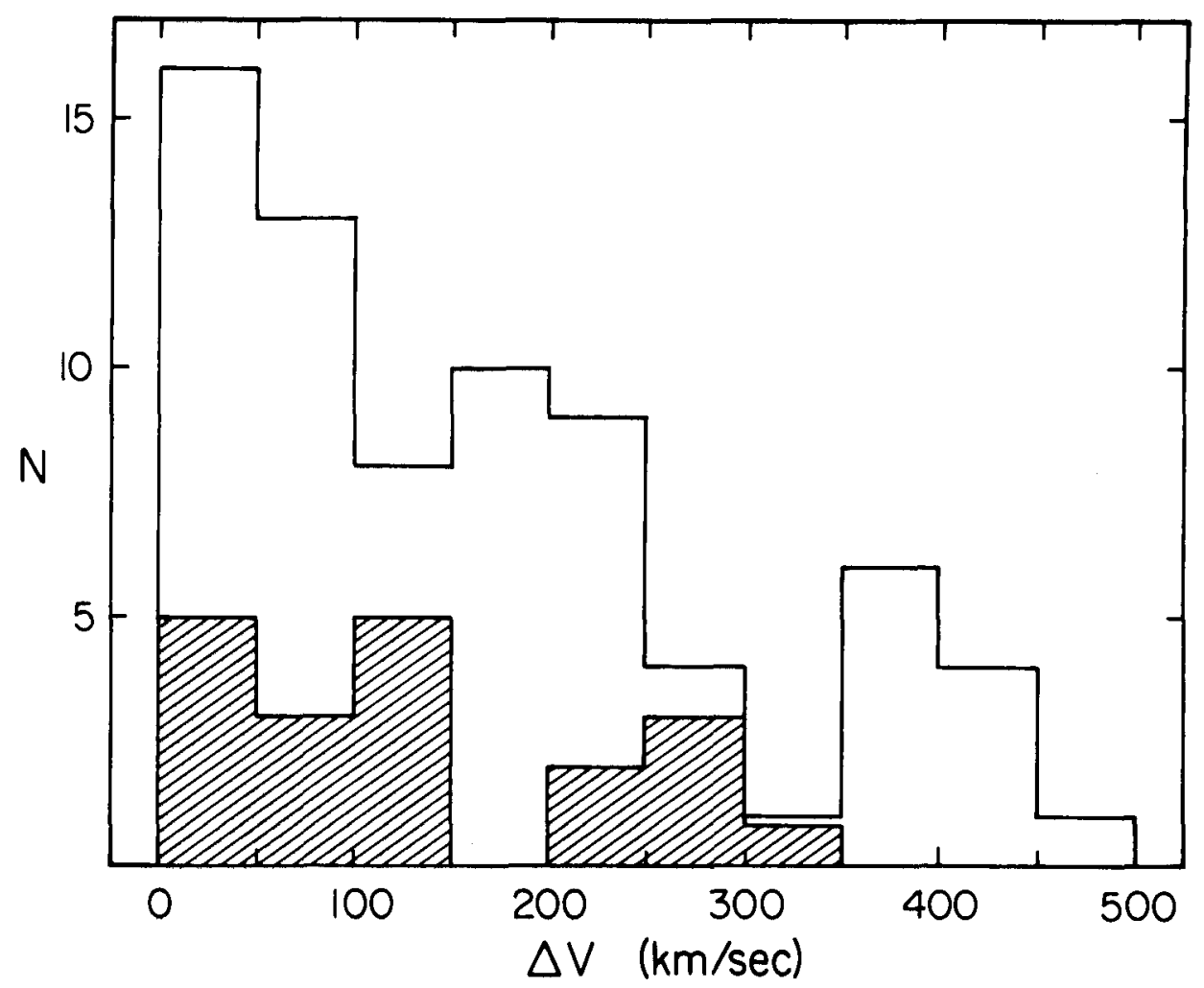

Figure 2. The distributions of velocity differences (in $\mathrm{km}$ sec $^{-1}$ for Turner's (1975) field galaxy pairs and Markarian pairs (shaded).

For the comparison of mass to light ratios, we use the ratio defined by equations (1) and (2). Table 2 gives the arithmetic mean of this parameter and its one sigma mean erior intervals for Turner's spiral and irregular (OS, S, I) sample, Turner's complete sample and for both the selected sample of Markarian binaries and the sample of 19 pairs or doubles (excluding 211-212).

TABLE 2 MEAN MASS TO LIGHT RATIOS

\begin{tabular}{lcc}
\hline SAMPLE & $\begin{array}{l}\text { Number of } \\
\text { Galaxies }\end{array}$ & Mean M/L \\
\hline FIELD GALAXIES & & $21(14-28)$ \\
OS, S, I pairs & 40 & $23(16-30)$ \\
AlI pairs & 73 & \\
MARKARIAN GALAXIES & & $19(3-34)$ \\
Selected pairs & 13 & $22(10-34)$ \\
All pairs & 19 & 22 \\
\hline
\end{tabular}


Contrary to the claim of Heidmann and Kalloghlian (1973), the Markarian binaries are not unbound and violently flying apart, and, in fact, appear to have the same $\langle M / L\rangle$ as the field spiral and irregular binaries. A comparison of the distribution of individual $M / L$ values for the Markarian and Turner binary galaxies shows that both samples are probably drawn from the same parent population of separations and velocity differences. The actual value of the mean $M / L$ for Markarian binaries, after correction for selection and orbital effects (Turner 1975), is then about 50 in solar units.

Heidmann and collaborators (Heidmann and Kalloghlian 1973, 1975; Casini and Heidmann 1975) find that Markarian galaxies tend to be associated with themselves and other galaxies. There is an excess of pairs with small separations over the number predicted by a random distribution of galaxies. We think that this can be interpreted simply as the tendency for galaxies to cluster. In order to test this hypothesis, we counted the observed number of Markarian galaxies with separations in 0.1 degree bins up to 5 degrees. In Figure 3 we plot the $\log$ of the ratio of this observed number to the number expected from a random surface distribution of galaxies. There is definitely an excess of pairs with small separations. However, we also plot the mean relationships derived from the power-law fits of Davis and Geller (1976) for the two point angular correlation coefficient of field galaxies. By their nature these relations are constrained not to go below zero. The Markarian galaxies are not clustered or paired any more strongly than ordinary field galaxies. In fact, this suggests that the Markarian galaxies are distributed in space in the same way as other galaxies. This is supported still further by the similarities of their luminosity functions (Huchra and Sargent 1973, Huchra 1976). 


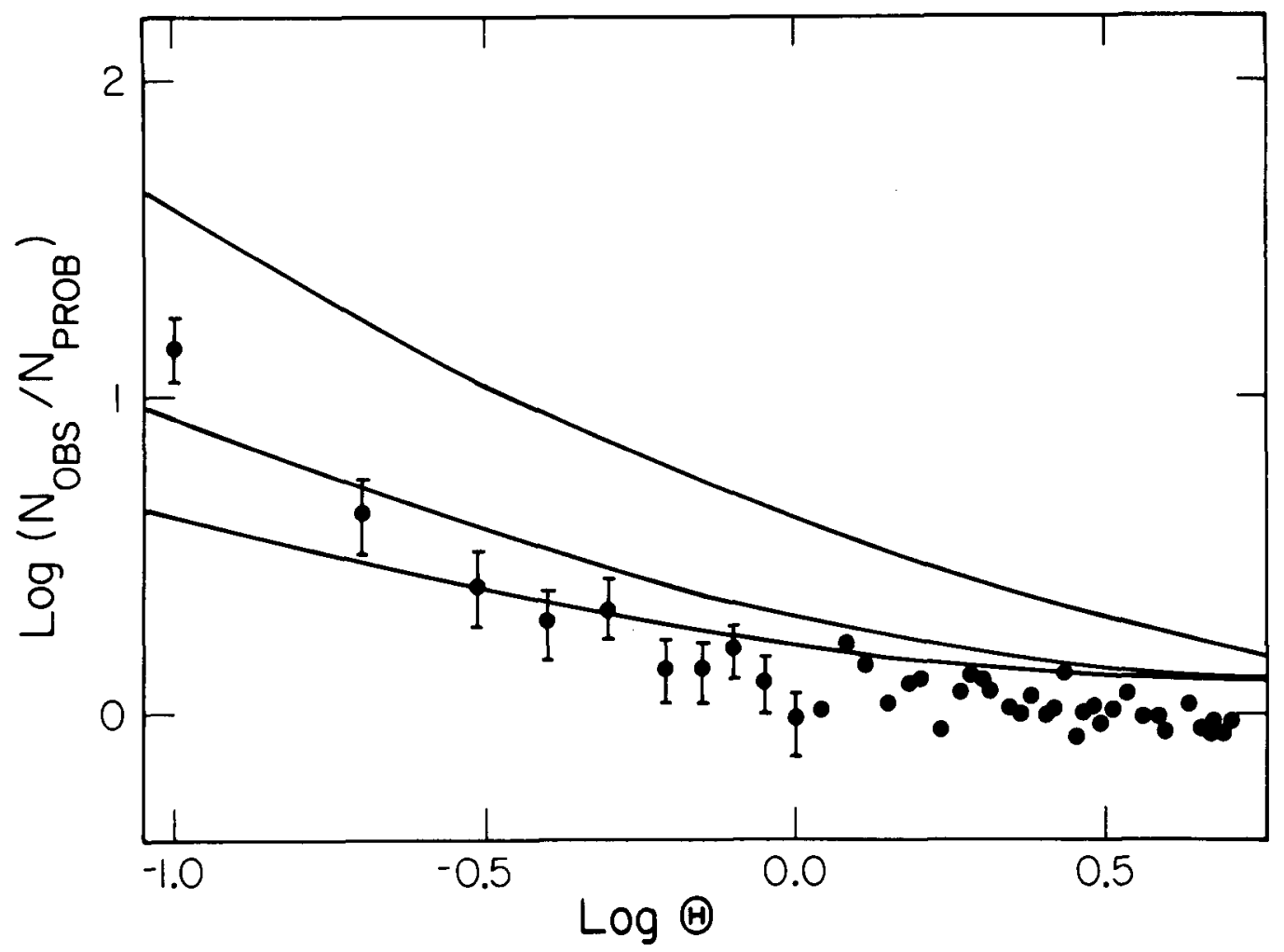

Figure 3. Logarithm of the number of observed pairs versus the number of pairs predicted by a random surface distribution as a function of angular separation. The circles are the data for all Markarian galaxies. The curves are the relationships derived by Davis and Geller (1976) for galaxies in the Nilson Catalogue. The upper line is for elliptical pairs, the lower line is for spiral-spiral pairs, and the middle line is for all types together.

Coupled with the similarity of mass to light ratios binding energies - for Markarian and field galaxy pairs, the clustering interpretation negates Heidmann's hypothesis of the "pair production" of Markarian galaxies. 


\section{REFERENCES}

Afanasjev, V., Karachontsev, I., and Notni, P. 1975, Astr. Nachr., 226, 233.

Arakelian, M. A., Dibai, E. A., and Esipov, V. F. 1972, Astrofizica, 8, 33 .

Arakelian, M. A., Dibai, E. A., Esipov, V. F., and Markarian, B. E. 1971, Astrofizica, 7, 177.

Bury, K. 1975, Statistical Models in Applied Science (New York: Wiley and Sons).

Casini, C., and Heidmann, J. 1975, Astr. and Ap. , 32, 127. Davis, M., and Geller, M. 1976, preprint. Denisyuk, E. K. 197la, Astr. Tsirk, 615, 4.

- 1971b, Astr. Tsirk, 624, 1.

- 1974, Astr. Tsirk, 8Q2, 1 .

Denisyuk, E. K., Babkin, I., and Sinyaeva, N. 1974, Astr. Tsirk, 837, 2 .

de Vaucouleurs, G., and de Vaucouleurs, A. 1964, Reference

Catalogue of Bright Galaxies (Austin: University of Texas

Press).

Heidmann, J., and Kalloghlian, A. T. 1973, Astrofizica, 2, 71. -1975, Astrofizica, 11, 229.

Huchra, J. 1976, ir preparation.

Huchra, J., and Sargent, W. L. W. 1973, Ap. J., 186, 433.

-1976, in preparation.

Noether, G. E. 1967, Elements of Non-Parametric Statistics

(New York: Wiley and Sons).

Page, T. 1966, in Proceedings of the Sixth Berkeley Symposium

on Mathematical Statistics and Probability, ed. L. M. Le Cam,

J. Neyman, and E. L. Scott (Berkeley: University of California).

Sargent, W. L. W. 1970, Ap. J. , 152, 765.

- 1972, Ap. J., 17Z, 7.

Searle, L., and Sargent, W. L. W. 1972, Ap. J., 173, 25.

Turner, E. I. 1975, Ph.D. Thesis, California Institute of Technology. 


\section{DISCUSSION}

J. HEIDMANN: I am a little astonished by these results which were not known to me before. I need to study them in order to make a statement. Off hand, I remark that most pairs we found have their components closer than 0.1 degree.

J.C. PECKER: I had the feeling, from differences of magnitudes between two "Markarian" galaxies, (and of the Heidmann-Kalloghlian study), that they form very homogeneous groups, showing a common nature, hence possibly a common origin. Indeed, I was quite convinced by Heidmann and Kalloghlian's paper. This remark is in regard to how the "sampling" is done.

J.P. HUCHRA: These Markarian pairs are not a homogeneous group in either magnitudes (of galaxies in the pairs) or morphological classifications.

V. RUBIN: Do you have enough velocity information to make any statement about the alignment of spin-axes for these pairs?

J.P. HUCHRA: NO.

E.M. BURBIDGE: What is the separation of the pair that you said was not a pair - with a very large difference of velocity?

J.P. HUCHRA: 3 arc minutes.

G.DE VAUCOULEURS: Your study confirms that Markarian galaxies are a heterogeneous collection of interesting objects, not a special class or a new type.

J.P. HUCHRA: Yes, in fact the finding of all facets of my investigation has been that the Markarian galaxies that do not exhibit extremely peculiar spectroscopic properties, as do the Markarian Seyferts and QSOs, are no different from the bluer field galaxies. They have a wide variety of photometric and morphological properties.

L. GOUGUENHEIM: From a $21-\mathrm{cm}$ line study, made at Nancay, of Markarian galaxies belonging to the subclass of non-dwarfs with narrow emission lines it appears that most of these galaxies could be classical galaxies of the Hubble sequence. They appear only as being bluer and more luminous than expected for their type: 
J. HEIDMANN: I would like to add that we never said that all Markarian pairs are unbound. Using the $21-\mathrm{cm}$ line, we obtained very good data for two pairs which, in order to be bound, would require very high $\mathrm{M} / \mathrm{L}$ ratios: 505 for Ma 7-8 and 230 for Ma 56-57 (Bottinelli, Duflot, Gouguenheim, Heidmann, 1975, Astron. Astrophys. 41, 61). In that same paper we even show that the pair Ma 325-326 may be bound.

J.P. HUCHRA: I do not think Markarian's 7 and 8 are a physical pair, and their separation of almost $1 / 2$ degree puts them out of my sample.

W.G. TIFFT: Is there any homogeneity of emission line properties in your sample? If sorted according to emission line strength did you find any differences in other properties?

J.P. HUCHRA: There is a wide variety of emission line properties in this sample, from purely absorption line systems to strong, sharp emission lines. I have not tried to sort the systems by spectroscopic properties. In the non-Seyfert Markarian galaxy sample as a whole, there are correlations between color, emission line strength and absolute magnitude, with the intrinsically fainter systems being bluer and having stronger emission and excitation.

E. SCOTT: 1) Will you comment on your corrections for the separation cut off? The corrections had a large effect, you say, from about 20 to 50 , but you did not say what you did.

2) Have you compared your distributions of ratios with those of Page and other earlier (Holmberg)?

3) I do not believe that a test, such as rank sum, will indicate no difference in the distributions (keep in mind that "no difference" $\neq$ "same" with such tiny samples). The rank sum test is not the best non-parametric test for comparing two distributions such as you are doing. Use Kolmogorov-Smironov test.

4) You are not justified in discarding a pair just because $\Delta v$ is large. Since distribution of $\Delta v$ is skew, it is "outlier prone".

J.P. HUCHRA: 1) I used the same selection criteria (ie. separation and nearest neighbor) as E. Turner, and the corrections for selection and projection were taken from his derivations using a much larger sample. 
2) No, both the Turner sample and this were selected in the same way so I thought that this was the most reasonable sample.

3) The test does, it shows that the distributions of $\Delta v, \mathrm{~d}_{\mathrm{kpc}}$ and $M / L$ are not different for the two samples. I think the rank-sum test was adequate for the data.

4) This may be true.

T. JAAKKOLA: I have made a study of the magnitude-redshift relation within pairs of Zwicky's compact galaxies (part of which are Markarian galaxies). Especially in blue pairs, the fainter components appear systematically redshifted with respect to the brighter ones. The same is the case for pairs of normal galaxies (Jaakkola, Proc. III Europ. Astron. Meeting, Tbilizi). The positive $(\mathrm{m}, \mathrm{z})$-relation within pairs indicates that either there are many optical pairs included, or the pairs are disrupting or, which is most probable on my personal weighting, the fainter components have excess non-velocity redshifts. In any case it is improper to use the virial theorem for determination of $\mathrm{M} / \mathrm{L}$ in these systems.

J.P. HUCHRA: I do not think there is a correlation of redshift and magnitude in this sample, but I have not checked.

G. BURBIDGE: Why did you reject the only pair which has a redshift difference such that it would clearly be unbound if you included it?

J.P. HUCHRA: It may be true that this is a discrepant redshift. However, on the basis of a random distribution, you would expect a few chance superpositions in this sample. Following in the footsteps of previous workers, I have discarded this as not being a physical pair, and I do not think this is unreasonable. In any case, the rest of the sample are the same as the normal galaxies. 